\title{
A Rare Case of Chylothorax due to Pulmonary Lymphangiectasia in a 7-Year-Old Boy
}

\author{
Giovanni Ruggeri ${ }^{1}$ Francesca Destro ${ }^{1}$ Michela Maffi ${ }^{1}$ Giulio Gregori ${ }^{1}$ Mario Lima ${ }^{1}$ \\ ${ }^{1}$ Department of Pediatric Surgery, S. Orsola-Malpighi Hospital, \\ Bologna, Italy \\ Eur J Pediatr Surg Rep 2013;1:18-20. \\ Address for correspondence Mario Lima, MD, Department of Pediatric \\ Surgery, S. Orsola-Malpighi Hospital, Via Massarenti 11, Bologna \\ 40138, Italy (e-mail: mario.lima@unibo.it).
}

\author{
Abstract \\ Keywords \\ - pulmonary \\ lymphangiectasia \\ - chylothorax \\ - thoracoscopy
}

Pulmonary lymphangiectasia (PL) is a rare condition characterized by dilatation of the lymphatic vessels. Post-neonatal PL is usually associated with pleural effusion and should therefore be suspected in the presence of chylothorax. We describe a post-neonatal manifestation of PL in a 7-year-old boy presenting chylothorax. Radiological examinations included thorax X-ray, ultrasound, and computed tomography scans. After the failure of conservative management (maintenance of the chest tube, total parenteral nutrition, administration of somatostatin synthetic analogues) we performed a thoracoscopic massive ligation of the thoracic duct's collateral along with a lung biopsy. Histology was compatible with type 1 congenital pulmonary lymphangectasia. One month after surgery a thoracoscopic pleurodesis was required for persistent chylothorax. The boy is now doing well 1 year after surgery.

\section{Introduction}

Pulmonary lymphangiectasia (PL) is a rare disease characterized by dilated but otherwise normal lymphatics typical of infancy and early childhood. The postneonatal form of PL has a better prognosis, ${ }^{1,2}$ but it can be misdiagnosed with other respiratory diseases. We report a rare case of PL presenting in a 7-year-old healthy boy with chylothorax, effectively treated with mini-invasive surgery.

\section{Case Report}

A 7-year-old boy came to our attention for suspected chylothorax. Up to 2 months before when he was treated for Streptococcus pyogenes septic shock at another hospital, the boy had always been well. The shock was probably due to bilateral pneumonia as shown by radiological investigations. In that occasion, he needed respiratory support (orotracheal ventilation for 10 days). After the positioning of a pleural drainage together with the administration of antibiotic therapy, there was a progressive improvement of clinics, laboratory tests, and radiological examinations so he was discharged. During radiological follow-up (2 months later), an organized left pleural effusion was shown by thorax ultrasound (US). Another chest drain was placed and the patient was transferred to our pediatric surgery unit. The milky leakage had the chemical features of chilo: triglycerides $>110 \mathrm{mg} / \mathrm{dL}$, protein $=5.7 \mathrm{~g} / \mathrm{dL}$, lactate dehydrogenase $=290 \mathrm{U} / \mathrm{L}$, glucose $=77 \mathrm{mg} / \mathrm{dL}$, amylase $=37 \mathrm{U} / \mathrm{L}$, albumin $=3.7 \mathrm{~g} / \mathrm{dL}$, cellularity $>1,000$ cell $/ \mathrm{L}$ (lymphocytes $90 \%$, neutrophils $2 \%$, and macrophages $8 \%$ ).

We performed thorax X-ray and computed tomography (CT) with the evidence of left pleural effusion, small left apical and medial pneumothorax, thickening of interlobular septa and bronchial wall on both sides (left $>$ right), and areas of ground-glass attenuation ( - Fig. 1).

Initial therapy was conservative. The chest tube was maintained for the drainage of effusions, a central line was placed for total parenteral nutrition, antibiotic (ceftriaxone) and somatostatin synthetic analogues (octreotide, $1 \mu \mathrm{g} / \mathrm{kg} /$ $\mathrm{h}=20 \mu \mathrm{g} / \mathrm{h}$ gradually increased up to $5 \mu \mathrm{g} / \mathrm{kg} / \mathrm{h}=100 \mu \mathrm{g} / \mathrm{h}$ ) were used as pharmacological agents. Overall, this management was well-tolerated except for a few presyncope. Differential diagnoses included traumatic events (no attributable received

October 21, 2012

accepted after revision

November 26, 2012

published online

March 19, 2013 (c) 2013 Georg Thieme Verlag KG Stuttgart · New York
DOI http://dx.doi.org/ 10.1055/s-0033-1337111. ISSN 2194-7619. 


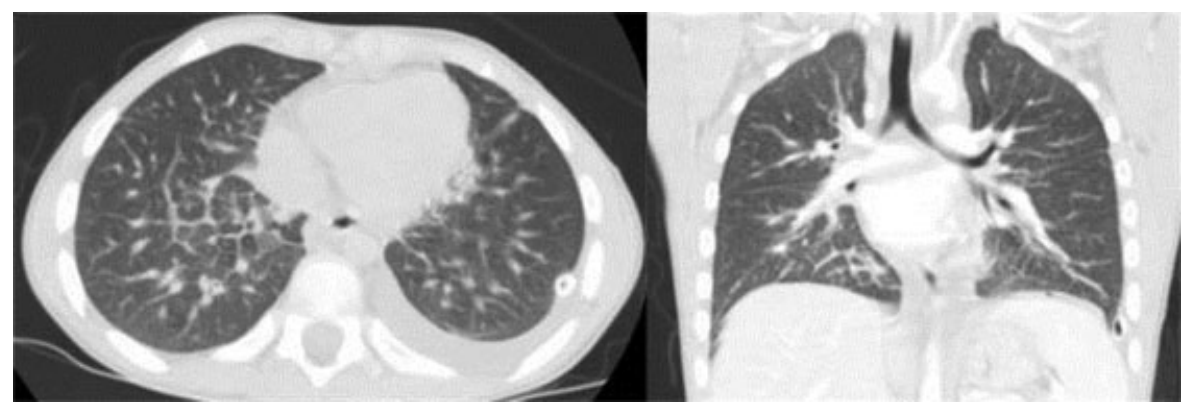

Fig. 1 Thorax computed tomographic scans showing left pleural effusion, ground-glass phenomenon, and thickening of bronchi and interlobular septa.

anamnesis), neoplasm (bronchoalveolar lavage negative for malignant tumor cells and CT negative for suspected masses), heart diseases (normal echocardiogram), alterations of large vessels (a Doppler US was used to rule out occlusive processes), storage diseases (negative US and laboratory tests), and Gorham-Stout syndrome (normal plain radiographs). Blood tests never showed an alteration of inflammatory indexes. Plasma transfusions and administration of albumin were required for progressive loss of protein. After 1 month, we decided to refer the patient for surgery because there was no significant reduction of the effusion (mean fluid drained: $818 \mathrm{~mL} / \mathrm{d}$ ). Before surgery, the patient was given $200 \mathrm{cc}$ of cream to highlight the origin of the leak more easily. An endobronchial blocker was used to exclude the left lung. We used three operative trocars and a $5-\mathrm{mm}$ optic with the patient lying in the standard right lateral decubitus with slightly pronation. The pleural space was occupied by some adhesions that were detached. Visceral pleura appeared suffering and macerated (-Fig. 2).

We found a poorly defined secreting area near the posterior mediastinum. We performed a massive ligation of the thoracic ducts collaterals applying metal clips. After obtaining a sharp reduction of the leak, we took a lung biopsy and we left two pleural tubes. Histological examination revealed type 1 congenital pulmonary lymphangiectasia ( - Fig. 3 ).

Postoperatively, the boy was doing well. After 6 days, he was discharged with pharmacological decalage. Three weeks later, there was an increment of pleural effusion on a chest $\mathrm{X}$-ray and so the boy was readmitted. We performed thoracoscopic mechanical and chemical pleurodesis with scissors, hook coagulator, and talc. At the end of the maneuver, we left two pleural tubes. The boy required intensive postoperative



Fig. 2 Thoracoscopy permits the visualization of suffering visceral pleura (left) and a secreting area near the mediastinum (center). A massive ligation of lymphatic vessels with metal clips reduces the effusion (right).


Fig. 3 Lung biopsy. The microscopic examination shows lymphatic cystic dilatations in the visceral pleura (left), surrounding the alveoli. In the lung parenchyma, cystic lymphatic areas are identified with CD31 staining, specific for endothelial cells (right). 
care for 9 days and respiratory support for the onset of a left pneumothorax. The pleural tubes were removed respectively 7 and 14 days after surgery (mean fluid drained $=5 \mathrm{~mL} / \mathrm{d}$ ). Enteral nutrition was gradually reintroduced and the overall hospital stay after surgery was 25 days. During the follow-up, we performed a thorax X-ray every month for 5 months and then every 2 months. Radiological investigations showed great improvement of both the pleural and parenchymal components. The boy was doing well 1 year after surgery.

\section{Discussion and Conclusions}

Pulmonary lymphangiectasia (PL) is a rare developmental disorder with diffuse dilatation of the pulmonary lymphatics of unknown origin that led to fluid accumulation. Noonan et $\mathrm{al}^{3}$ classified the disease into the primary form (congenital), that can be generalized, localized (pulmonary), or associated with syndromes (syndromal-Noonan, Ullrich/Turner, Down) and the secondary form (due to pulmonary hypertension, cardiovascular problems, or lymphatic obstruction). The overall incidence is unknown with men being affected more than women in the primary form. ${ }^{4}$ Symptoms can arise prenatally (hydrops fetalis and polyhydramnios), at birth (cyanosis, tachypnea, respiratory distress) or, rarely, after a period of wellness in stable patients with localized forms. Postneonatal congenital PL is commonly associated with pleural effusions and chylothorax. ${ }^{5,6}$ Chylothorax is associated with high mortality and morbidity if not treated. It is characterized by specific chemical features of the pleural effusion that has a high triglyceride content with elevated protein and albumin concentration and a cell count of more than 1,000 cells/mcL with a predominance of lymphocytes $(>80 \%){ }^{7}$ Chylothorax can be the only sign of PL. Radiological examinations are useful to point out elements important for diagnosis ${ }^{8}$ and to verify improvements or to detect relapses during the follow-up. In our case, CT scans showed inhomogeneity of the lung parenchyma with a diffuse thickening of the septa surrounding the lobules (Kerley B septal lines) and patchy areas of ground-glass infiltrates. The high resolution CT is the radiological technique of choice for diagnosing PL, although only the lung biopsy identifies specific features of this disease. The treatment of choice is the conservative one for the support of the patient: pleural drain, total parenteral nutrition, and administration of octreotide. ${ }^{1,5}$ Chest tubes are required in patients with rapidly expanding pleural effusion. ${ }^{5}$ Parenteral nutrition and formula rich in medium chain triglycerides is important in the reduction of lymphatic production. Nonunivocal data are available regarding the use of octreotide. ${ }^{9}$ We decided to use it intravenously but the increment of the doses was limited by the rise of syncopes. We planned to monitor vital parameters (pressure and frequency rate) and glucose levels every 4 hours to identify and avoid the drug's collateral effects. During the treatment, the patient was also given plasma and albumin that he had lost with the large amount of fluid drained. Surgery should be considered after 2 to 4 weeks of conservative management if the patients remain stable. ${ }^{10}$ Some authors suggest to limit the length of the conservative treatment stating that its efficacy is related to the effusion drained during the first few days. ${ }^{11}$
Actually no prognostic factors have been identified yet. As suggested by Beghetti et $\mathrm{al}^{10}{ }^{10}$ we used a significant cutoff below $10 \mathrm{~mL} / \mathrm{kg} /$ die of fluid drained as indicator of success. Surgery is required when pleural effusions persist (intractable chylothorax). In literature, various surgical techniques are proposed: direct ligation of the thoracic duct (if the point of rupture is readily identified), pleurodesis, pleurectomy, position of pleuro-peritoneal shunts. ${ }^{12,13}$ On the basis of our experience, we decided to treat the patient with a thoracoscopic approach that was useful both for diagnosis and therapy and produces all the advantages of a mini-invasive surgery. Considering the possible complications of talc pleurodesis (most of all pneumonitis and chest pain) and the absence of a precise diagnosis, we decided to avoid a nonreversible maneuver preferring massive ligation. Actually, the daily drain was really abundant and there was no evidence of a precise leakage point, so that the talc instillation could also be considered as a first approach.

Long-term outcome of patients affected by PL remain uncertain. ${ }^{4}$ In most cases, symptoms gradually improve over time, especially in forms with later onset (childhood).

\section{Conflict of Interest}

None

\section{References}

1 Esther CR Jr, Barker PM. Pulmonary lymphangiectasia: diagnosis and clinical course. Pediatr Pulmonol 2004;38(4):308-313

2 Barker PM, Esther CR Jr, Fordham LA, Maygarden SJ, Funkhouser WK. Primary pulmonary lymphangiectasia in infancy and childhood. Eur Respir J 2004;24(3):413-419

3 Noonan JA, Walters LR, Reeves JT. Congenital pulmonary lymphangiectasis. Am J Dis Child 1970;120(4):314-319

4 Mettauer N, Agrawal S, Pierce C, Ashworth M, Petros A. Outcome of children with pulmonary lymphangiectasis. Pediatr Pulmonol 2009;44(4):351-357

5 Bellini C, Boccardo F, Campisi C, Bonioli E. Congenital pulmonary lymphangiectasia. Orphanet J Rare Dis 2006;1:43

6 Xiao ZY, Tao Y, Tang XY, Chen GJ, Guo L. Congenital pulmonary lymphangiectasis. World J Pediatr 2009;5(1):68-70

7 Soto-Martinez M, Massie J. Chylothorax: diagnosis and management in children. Paediatr Respir Rev 2009;10(4):199-207

8 Nobre LF, Müller NL, de Souza Júnior AS, Marchiori E, Souza IV. Congenital pulmonary lymphangiectasia: CT and pathologic findings. J Thorac Imaging 2004;19(1):56-59

9 MacLean JE, Cohen E, Weinstein M. Primary intestinal and thoracic lymphangiectasia: a response to antiplasmin therapy. Pediatrics 2002;109(6):1177-1180

10 Beghetti M, La Scala G, Belli D, Bugmann P, Kalangos A, Le Coultre C. Etiology and management of pediatric chylothorax. J Pediatr 2000; 136(5):653-658

11 Graham DD, McGahren ED, Tribble CG, Daniel TM, Rodgers BM. Use of video-assisted thoracic surgery in the treatment of chylothorax. Ann Thorac Surg 1994;57(6):1507-1511, discussion 1511-1512

12 Wurnig PN, Hollaus PH, Ohtsuka T, Flege JB, Wolf RK. Thoracoscopic direct clipping of the thoracic duct for chylopericardium and chylothorax. Ann Thorac Surg 2000;70(5):1662-1665

13 Vargas FS, Milanez JR, Filomeno LT, Fernandez A, Jatene A Light RW. Intrapleural talc for the prevention of recurrence in benign or undiagnosed pleural effusions. Chest 1994;106(6): 1771-1775 